\title{
COMPUTERS IN CLINICAL PRACTICE
}

\section{Terry Hannan, Consultant Physician, Miranda, NSW}

T his article is a brief introduction to the benefits of computers for patient care in clinical practice. The benefits derived from automation of the administrative components of medical practice are well documented and are not addressed in this article.

Modern health care is characterised by an extensive data/information overload which is a direct consequence of the technology supporting the delivery of health care. The number of new laboratory procedures continues to rise between 5 per cent and 10 per cent a year, resulting in additional data/information to be processed in decisionmaking. The limitations of the human mind to process large amounts of information without making errors are established and have been shown to occur in medical and non-medical environments. Airline pilots have been shown to make significant errors in decision-making when the information load is large or when 'random noise', i.e. unexpected data input, occurs. The use of computer technology to reduce these errors is a major reason that flying is such a safe activity ${ }^{1}$. In the clinical environment, individuals who make decisions about patient care can be confronted with up to 50,000 data items or more in the short time available. We often disguise our deficiencies in decision-making under the labels of 'clinical judgment', 'intuition', and 'experience'.

In family practice or ambulatory care, the information load is less than in hospitals but remains at a level where the limitations on information processing still occur. In these environments, data and information are described as being 'granular'. The data are often collected during patient encounters which are widely separated over time and the health care practitioner has no tools for integrating the disseminated data in a timely, reliable manner ${ }^{3}$.

Consequences of the data/information overload in health care delivery are:

ㅁ

increased costs of health care;

inappropriate variation in health care;

reduced quality of care;

reduced improvement in patient outcomes;

decreased compliance with the standards of care; and

in some cases, negligent care ca, $^{45,6}$

The medical record remains the cornerstone for the communication of health care. Information in the current manual record is fragmented and often illegible, and there is often no standardisation for recording data. Data are poorly accessible and do not provide adequate feedback for decision support. In relation to medical record security, Robert Esterhay MD, from Bethesda, Maryland, recently stated that "the joke amongst hospital administrators was the inpatient chart was the most secure information system in existence, because not even the house staff and attending physicians knew where to find all the information on a particular patient"

Despite the limitations of the current medical record we continue to use it to manage our patients, often not

\section{TABLE 2}

CLINICAL TASKS GENERATING COMPUTER REMINDERS

Computer-recommended action

Stool occult blood

Cervical smear

Hemafocrit/Haemoglobin

Chest $x$-ray

Pneumococcal vaccine

Tuberculosis skin test

Serum potassium

Mammogram

Influenza vaccine

Diet

Reticulocyte count

Total iron binding

Digitalis

Liver enzymes

Antacids

recognising its incapacity to act as an effective tool for supporting health care.

In the 1960 s and early 1970 s researchers began investigating the possible benefits of computer technology for the management of medical information in patient care. In 1976 McDonald from the Regenstrief Health Centre, Indianapolis, published the results of one of the most important studies on the use of computers in patient care. He confirmed that clinical errors could be reduced by prospective computer suggestions about simple clinical events based on recorded patient data. McDonald hypothesised that many medical errors occur because of the physician's intrinsic limitations as an information processor rather than because of remediable flaws in the individual's fund of knowledge. In the conclusions to his study McDonald demonstrated that ignorance of medical facts contributed very little to these deficits. The main reason for error in patient care was that "the amount of data presented to the physician per unit time is more than he can process without error. The computer augments the physician's capabilities and therefore reduces his error rate ... it is very likely that the physicians in these studies were simply unable to detect all the multitudinous conditions specified by the standards used". The physicians studied were regularly unable to comply with the standards of care they had themselves defined.

The Regenstrief group subsequently extended the use of computer-generated reminders to the domain of preventive care. Using the "introspective" facility of the computerised medical record, which applied clinical rules to stored patient data, the system produced prompts, reminders or alerts for the clinician who then was able to judge whether the computer-generated recommendation was appropriate. The computer is able to process the clinical data rapidly (this is normally a tedious task for humans) and then display the relevant data/information in a summary format relevant to the tasks being undertaken. Table 2 lists the 15 clinical situations in which reminders were used in the study. 


\section{Computers in clinical practice}

Continued from page 11

The study found that clinicians who used the computergenerated reminders implemented preventive care measures twice as often as those in the control group who had only standard paper medical records for accessing the patients' clinical information. On average, clinicians chose to ignore them in 50 per cent of cases overall, and for one reminder ignored them in 92 per cent of cases. The authors concluded: "Physicians seem well able to resist suggestions with which they disagree" $"$. The results appeared to confirm that the physicians in the study produced errors of omission and the computer reminders improved the correspondence between their actions and intentions. No overall benefit was detected in patient outcomes. However, the sample size and study duration were not large enough to detect changes in patient outcomes. For the pneumococcal and influenza vaccine groups there was a reduction in winter morbidity, suggesting improved outcomes.

The low compliance with certain reminders was interpreted as clinicians being slow to accept new clinical guidelines rather than the use of computer-generated reminders as clinical aids.

The availability of computerised medical record systems like the Regenstrief Medical Record System should encourage physicians to use more preventive care measures, thus providing more effective patient care and a reduction in health care costs. In 1993 Fries et al reviewed mechanisms for reducing health care costs through a reduction in the demand for medical services. The authors stated that "preventable illness makes up approximately 70 per cent of the burden of illness and the associated costs, and accounts for eight of the nine leading categories and for 980,000 deaths per year". ${ }^{10}$

Some of the potential benefits of such systems were confirmed in a study published in 1993 from the 350-bed Regenstrief Health Centre in Indianapolis. Here the use

\section{TABLE 3}

REDUCTIONS IN CLINICAL UTILISATION [REGENSTRIEF HEALTH CENTRE(a)]

\begin{tabular}{|lr|}
\hline Parameter & \% Reduction \\
\hline Test ordering & 12.5 \\
Bed charges & 11.9 \\
Medications & 15.3 \\
Length of stay & 10.5 \\
Other & 15.2 \\
\hline
\end{tabular}

(a) Source: ref 11.

of the computerised medical record system led to significant improvement in markers of health care delivery, mainly through a reduction in the use of clinical resources (Table 3 ).

1. Drinkwater BL. Performance of civil aviation pilots under conditions of sensory input overload. Aerosp Med 1967; 38:164-8.

2. Weed L. New premises and tools for medical care and medical education. Proceedings - International Symposium on Medical informatics and Education, University of Victoria, Victoria, BC. 1989; 19-22.

3. Blum BI. Clinical Information Systems. New York, Springer-Verlag. 1986

4. Johns RJ, Blum BI. The use of clinical information systems to control costs as well as improve patient care. Trans Am Clin Assoc 1973; 90: 104-152.

5. Wennberg $J$, Gittelsohn A. Small area variations in health care delivery. Science 1973; 182:1102-8.

6. Brennan TA et al. Incidence of adverse events and negligence in hospitalised patients - results of the Harvard Medical Practice Study I. New Engl J Med 1991; 324(6):370-376.

7. AMIA Spring Congress, St Louis, 1992.

8. McDonald CI. Protocol-based computer reminders, the quality of care and the non-perfectibility of man. New Engl J Med 1976; 295:1351-5. 9. McDonald CJ, Hui SL, Smith DM, Tierney WM, Cohen SJ,

Weinberger M, McCabe GP. Reminders from an introspective computer record. Ann Int Med 1984; 100:130-8.

10. Fries JF et al. Reducing health care costs by reducing the need and demand for medical services. New Engl J Med 1993; 329(5):321-325.

11. Tierney WM Miller ME Overhage JM, McDonald CJ. Physician inpatient order writing on microcomputer workstations - effects on inpatient order writing on microcomputer work
resource utilisation. JAMA 1993; 269:379-383.

\section{PUBLIC HEALTH EDITORIAL STAFF}

The editor of the Public Health Bulletin is Dr Michael Frommer, Director, Research and Development, NSW Health Department; production manager is Dr Marie-Louise Stokes, and assistant editor is Dr Valerie Delpech.

The Bulletin aims to provide its readers with population health data and information to motivate effective public health action. Articles, news and comments should be 1,000 words or less in length and include a summary of the key points to be made in the first paragraph. References should be set out using the Vancouver style, the full text of which can be found in British Medical Journal 1988; 296:401-5.

Please submit items in hard copy and on diskette, preferably using WordPerfect 5.1, to the editor, NSW Public Health Bulletin, Locked Mail Bag 961, North Sydney 2059. Facsimile (02) 3919232.

Please contact your local Public Health Unit to obtain copies of the NSW Public Health Bulletin. 